\title{
BMJ Open Cost-effectiveness of rivaroxaban compared with enoxaparin plus warfarin for the treatment of hospitalised acute deep vein thrombosis in China
}

\author{
Li Yang (D) , ${ }^{1}$ Jingjing $\mathrm{Wu}^{2}$
}

To cite: Yang L, Wu J. Costeffectiveness of rivaroxaban compared with enoxaparin plus warfarin for the treatment of hospitalised acute deep vein thrombosis in China. BMJ Open 2020;10:e038433. doi:10.1136/ bmjopen-2020-038433

- Prepublication history for this paper is available online. To view these files, please visit the journal online (http://dx.doi. org/10.1136/bmjopen-2020038433).

Received 11 March 2020 Revised 27 May 2020 Accepted 29 May 2020
Check for updates

(c) Author(s) (or their employer(s)) 2020. Re-use permitted under CC BY-NC. No commercial re-use. See rights and permissions. Published by BMJ.

${ }^{1}$ School of Public Health, Peking University, Beijing, China ${ }^{2}$ Bayer Healthcare Company Ltd, Beijing, China

Correspondence to

Professor Li Yang;

lyang@bjmu.edu.cn

\section{ABSTRACT}

Objective Limited economic evaluation data for rivaroxaban compared with standard of care $(\mathrm{SoC})$ exists in China. The objective of this analysis was to evaluate the cost-effectiveness of rivaroxaban compared with current SoC (enoxaparin overlapped with warfarin) for the treatment of acute deep vein thrombosis (DVT) in China. Methods A Markov model was adapted from a payer's perspective to evaluate the costs and quality-adjusted life years (QALYs) of patients with DVT treated with rivaroxaban or enoxaparin/warfarin. Clinical data from the EINSTEIN-DVT trial were obtained to estimate the transition probabilities. Data on Chinese health resource use, unit costs and utility parameters were collected from previously published literature and used to estimate the total costs and QALYs. The time horizon was set at 5 years and a 3-month cycle length was used in the model. A $5 \%$ discount rate was applied to the projected costs. One-way sensitivity analyses and probabilistic sensitivity analyses were undertaken to assess the impact of uncertainty on results.

Results Rivaroxaban therapy resulted in an increase of 0.008 QALYs and was associated with lower total costs compared with enoxaparin/warfarin (US\$4744.4 vs US\$5572.4, respectively), demonstrating it to be a cost-saving treatment strategy. The results were mainly sensitive to length of hospitalisation due to DVT on enoxaparin/warfarin, cost per day of hospitalisation and the difference in length of stay of rivaroxaban-treated and enoxaparin/warfarin-treated patients.

Conclusion Rivaroxaban therapy resulted in a cost saving compared with enoxaparin/warfarin for the anticoagulation treatment of patients with hospitalised acute DVT in China. Trial registration number NCT00440193; Post-results.

\section{INTRODUCTION}

Deep vein thrombosis (DVT) and pulmonary embolism (PE) together constitute venous thromboembolism (VTE) - a common disorder causing substantial disease burden and mortality globally. ${ }^{1}$ In China, the incidence of VTE (DVT and PE) is high among hospitalised patients, ${ }^{2} 3$ with incidence rate of 30.0, 8.7 and 3.0 per 100000 reported for DVT, PE and PE with DVT in a large

\section{Strengths and limitations of this study}

This study evaluated the cost-effectiveness of rivaroxaban for acute deep vein thrombosis treatment in China with a well-acknowledged and transparent method.

- This study could support the decision-making of stakeholders in China, including hospitals, payers and physicians.

- In this analysis, we set a lot of assumptions, in terms of patients' characteristics, inpatient setting and the treatment duration, which may limit the results being extrapolated to whole population.

- The utility data in the model were derived from literature and not specific to the Chinese population, which may impact the estimation of quality-adjusted life year.

epidemiological study in Chinese population. In addition, mortality rates of DVT, PE and PE with DVT were 9.0\%, $17.4 \%$ and $13.3 \%$, respectively. ${ }^{4}$ Consistent with this, VTE is among the major causes of death in hospitals. ${ }^{5}$ Clinical guidelines recommend the use of anticoagulant therapy to minimise the risk of mortality and VTE recurrence, with lowmolecular weight heparin (LMWH) overlapped with vitamin-K-antagonists (VKAs; mostly warfarin) being one of the current standard of care (SoC) ${ }^{6}$ However, there are several limitations to the SoC, for example, patients requiring injection, frequent international normalisation ratio (INR) monitoring and dose titrations, ${ }^{7}$ which result in unsatisfactory compliance and therapeutic outcomes in clinical practice. ${ }^{7}$

Rivaroxaban, an orally administered anticoagulant which does not require frequent monitoring or dose adjustments, ${ }^{8-10}$ when compared with enoxaparin plus warfarin (enoxaparin/warfarin), displayed similar efficacy and safety in preventing recurrent DVT and reducing the risk of bleeding events, as 
reported in the EINSTEIN-DVT trial. ${ }^{11}$ Evidence from several studies also suggests that rivaroxaban treatment results in a significant decrease in the number of hospitalisations and outpatient visits as well as a reduction in total hospitalisation costs. ${ }^{12} 13$

Although rivaroxaban has been approved for DVT treatment in China, its higher price ${ }^{14}$ compared with warfarin might be a barrier for some patients and payers. To address the concern of limited cost-effectiveness evidence for rivaroxaban and enoxaparin/warfarin in DVT, this study aimed to evaluate the cost-effectiveness of rivaroxaban versus enoxaparin/warfarin from a Chinese healthcare perspective based on findings of the EINSTEIN-DVT trial. $^{15}$

\section{METHODS}

A Markov model was developed to estimate the costeffectiveness of rivaroxaban compared with enoxaparin/ warfarin in the treatment of patients with acute DVT in hospitals, from the Chinese healthcare payer perspective, for a duration of 5 years. The duration was set based on the previous publication ${ }^{16}$ and clinical practice in China. The results of our study were reported using the Consolidated Health Economic Evaluation Reporting Standards. ${ }^{17}$

The patients evaluated in our model met the description of participants from the acute DVT arm of the EINSTEIN-DVT trial. ${ }^{15}$ All patients' age was set to 56 years at baseline as per the EINSTEIN study. The patients entered the model in the 'on-treatment' state and received oral rivaroxaban $(15 \mathrm{mg}$ two times per day for 21 days followed by $20 \mathrm{mg}$ per day) or enoxaparin $(1.0 \mathrm{mg} /$ $\mathrm{kg}$ subcutaneously for 8 days) plus warfarin (target INR of 2.0-3.0). Based on the perception that, in Chinese clinical practice, the actual anticoagulant treatment duration for patients with DVT is $<3$ months, the model assumed that all patients had received 3 months of anticoagulation treatment. The model also assumed that all patients received inpatient treatment in the acute phase, because the main risk factors of acute DVT events in China were prolonged immobilisation and malignant tumours ${ }^{18}$ and those patients were most likely to get treatment in inpatient setting when DVT was provoked.

The outcomes of the model included assessment of quality-adjusted life years (QALYs) and cost of treatment with rivaroxaban or enoxaparin/warfarin. Factors affecting the cost-effectiveness model were also determined. The model allowed tracking of patients with DVT through a standard treatment pathway and captured the common complications associated with DVT and its anticoagulant treatment. Probabilities of treatment discontinuation due to bleeding or non-compliance were also considered in the model. A 3-month cycle length with a 5 -year time horizon was used. Total medical costs were considered from a Chinese healthcare perspective and expressed as the 2017 USD exchange rate (US\$1=¥6.67), with future costs discounted at $5 \%$ per year.

\section{Model framework}

The Markov model was developed with 12 health states (figure 1) and presents progression between health states according to transition probabilities. The model also shows the estimates of life expectancy, health outcomes, resource use and cost of treatment. As per the model, patients were assumed to be on-treatment on initiation of either rivaroxaban or enoxaparin treatment after an index DVT event. Post-therapy, the patients may undergo several transition states, including acute bleeding

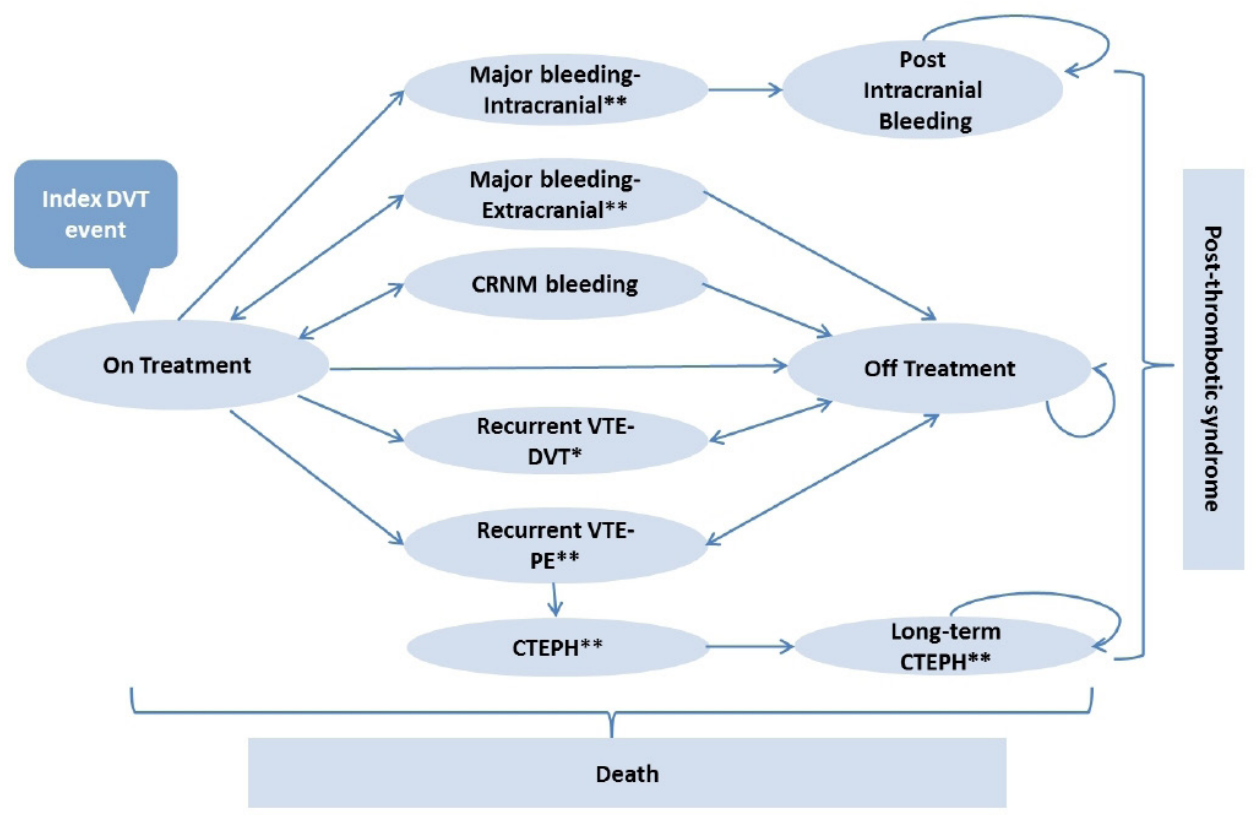

Figure 1 Model schematic. ${ }^{21}{ }^{*}$ DVT split into contralateral and ipsilateral. ${ }^{* *}$ Additional mortality. CRNM, clinically relevant nonmajor; CTEPH, chronic thromboembolic pulmonary hypertension; DVT, deep vein thrombosis; PE, pulmonary embolism; VTE, venous thromboembolism. 
events such as major intracranial (IC) bleeding, extracranial (EC) or clinically relevant non-major (CRNM) bleeding as well as recurrent VTE events (DVT or PE). The common long-term complications were considered in the model, including post-IC bleed state following IC bleed events, chronic thromboembolic pulmonary hypertension (CTEPH) after PE events and post-thrombotic syndrome (PTS) after DVT events. Recurrent DVT, risk of CTEPH and death were also considered in patients not receiving therapy. Each state was assigned a cost and utility weighting to calculate the total costs and QALYs of patients simulated in the model. ${ }^{19}$

\section{Model inputs}

\section{Core clinical data}

The clinical inputs used in the model, regarding the cost, safety and probability of events for both rivaroxaban and SoC, were obtained from the EINSTEIN-DVT study. ${ }^{15}$ The trial is a multicentre, randomised, open-label, eventdriven powered to show non-inferiority against warfarin. Total 3449 patients were included in the study: 1731 given rivaroxaban and 1718 given enoxaparin plus a VKA. The primary efficacy outcome was recurrent VTE and the principal safety outcome was major bleeding or CRNM.

For the time period of 0-3 months (cycle 1), event data for recurrent VTE, major bleeding (both IC and EC bleedings) and CRNM bleeding were considered as the baseline (table 1$).^{15}$ The probability of events with rivaroxaban in cycle 1 was inputted from the HR of rivaroxaban compared with enoxaparin/warfarin. Transition probabilities per cycle were calculated based on event risk. This was mainly derived from the EINSTEIN-DVT trial and other published literature. ${ }^{20-24}$

Risk of post-treatment events including recurrent VTE, bleeding, PTS, CTEPH and event-specific mortality rates in subsequent cycles were obtained from the published literature (table 1$)$.

\section{Discontinuation rates}

Based on findings from the EINSTEIN-DVT study, the model assumed that all patients with IC bleeding, $40 \%$ of patients with major EC bleeding and $11.3 \%$ of those with CRNM bleeding would discontinue the treatment. Complete discontinuation was assumed for patients with major bleeding events. However, for CRNM bleeding events, it was assumed that patients would discontinue therapy for 1 month only and treatment costs would be incurred for the remainder of the cycle.

\section{Utility inputs}

Utility values define health state associated quality of life with a range of $0-1(0=$ death and $1=$ best estimated health state). Evidence from published literature was used to determine the various utility values. The Chinese population norm value was taken as 0.929 (95\% CI 0.917 to 0.941), which was established in the landmark national EuroQol five dimensions questionnaire (EQ-5D) survey. ${ }^{25}$ This value was used as the basis for calculating the utilities of every health state. The utility value used for DVT was 0.884 (95\% CI 0.674 to 1.000 ), as demonstrated in the report by Locadia et al. ${ }^{26}$ Previous studies ${ }^{27}$ have reported increased treatment satisfaction with rivaroxaban compared with enoxaparin/warfarin; therefore, a disutility weight of 1.00 was assumed for rivaroxaban and a disutility value of 0.988 was assumed for enoxaparin/ warfarin. Utilities for other states were based on values in the previously published literature ${ }^{2628-31}$ (table 1).

\section{Resource utilisation and cost inputs}

On entry into the model, resource utilisation related to the index event (DVT) was used to analyse the difference between rivaroxaban and enoxaparin/warfarin, especially in terms of drug utilisation, monitoring frequency and hospitalisation. We assumed that patients received standard dosage and 3 months treatment in the absence of contraindications. It was also conservatively assumed that, in the first 3 months, patients receiving rivaroxaban would require three drug-monitoring visits and patients receiving enoxaparin/warfarin therapy would require eight visits. The length of stay (LoS) for hospitalised patients with DVT was set as 14.6 days (range 10.22-18.98 days) with enoxaparin/warfarin treatment ${ }^{7}$ and was assumed to be 3 days shorter with rivaroxaban therapy. ${ }^{32}$ Unit costs of rivaroxaban, enoxaparin and warfarin were based on local drug tariffs in China (table 1). The daily cost of hospitalisation was based on published literature (US\$363.65, range US\$254.55-472.74), ${ }^{33}$ with an average LoS of 14.6 days (range 10.22-18.98 days) for patients receiving enoxaparin/warfarin. ${ }^{7}$ The costs of managing the event were also based on the published literature ${ }^{33-35}$ and assumed to be equal across all treatment arms (table 1).

\section{Data analysis}

Data from published studies and assumptions from table 1 were used to calculate mean estimates of 5-year costs and QALYs for rivaroxaban and enoxaparin/warfarin. Basecase analyses-total costs and QALYs-were calculated for patients receiving rivaroxaban or SoC. Furthermore, the incremental cost-effectiveness ratio (ICER) was also calculated. Besides, we assumed a willingness-to-pay (WTP) threshold of US\$14 992.5 per QALY (ie, ¥100 000 originally in the model), which was less than three times the gross domestic product per capita in China in 2016 (US $\$ 24351.8^{36}$ ). An ICER of less than US $\$ 14$ 992.5 per QALY is then an indication that rivaroxaban is cost-effectiveness. ${ }^{37}$

To explore the effect of parameter uncertainty, we conducted one-way sensitivity analysis (OWSA) and probabilistic sensitivity analysis (PSA). In OWSA, the minimum and maximum estimates of clinical data, utility and costs were used in the model. For PSA, the variables were specified as distributions: the clinical input followed beta or normal distribution; costs inputs followed gamma distribution and utility data followed beta distribution. Then we run 1000 simulations in PSA to get 1000 estimates of 
Table 1 Model inputs

\section{Base case (lower-upper) Distribution Source}

Baseline events risk (0-3 months) - enoxaparin/warfarin

\begin{tabular}{|c|c|c|c|}
\hline rVTE & $2.6 \%(1.8 \%-3.3 \%)$ & Beta & EINSTEIN-DVT ${ }^{15}$ \\
\hline Probability that rVTE is DVT & $48.3 \%(37.8 \%-58.8 \%)$ & Beta & EINSTEIN-DVT ${ }^{15}$ \\
\hline Major bleeding & $0.9 \%(0.4 \%-1.3 \%)$ & Beta & EINSTEIN-DVT ${ }^{15}$ \\
\hline $\begin{array}{l}\text { Probability major bleeding is intracranial } \\
\text { bleeding }\end{array}$ & $12.5 \%(1 \%-24 \%)$ & Beta & EINSTEIN-DVT ${ }^{15}$ \\
\hline CRNM bleeding & $4.9 \%(3.9 \%-5.9 \%)$ & Beta & EINSTEIN-DVT ${ }^{15}$ \\
\hline
\end{tabular}

$\mathrm{HR}$-rivaroxaban versus enoxaparin/warfarin

$\begin{array}{llll}\text { rVTE } & 0.68(0.44-1.04) & \text { Log-normal } & \text { EINSTEIN-DVT }^{15} \\ \text { Major bleeding } & 0.65(0.33-1.30) & \text { Log-normal }^{15} & \text { EINSTEIN-DVT }^{15} \\ \text { CRNM bleeding } & 1.055(0.828-1.342) & \text { Log-normal }^{15} & \text { EINSTEIN-DVT }^{15}\end{array}$

Events risk-long-term complications

$\begin{array}{lcll}\text { rVTE (10year risk) } & 39.9 \%(35.4 \%-44.4 \%) & \text { Beta } & \text { Prandoni et al }^{20} \\ \text { Bleeding (subsequent cycles) } & 0 & - & \text { Assumption } \\ \text { Postintracranial bleeding } & 56.4 \% & - & \text { Linkins et al }^{21} \\ \text { CTEPH (2-year risk) } & 1.25 \%(1.14 \%-1.63 \%) & \text { Beta } & \text { Miniati et al }^{22} \\ \text { PTS (1-year risk) } & 18 \%(14.7 \%-21.3 \%) & \text { Beta } & \text { Prandoni et al }^{23}\end{array}$

Mortality

$\begin{array}{lccc}\text { PE } & 25.0 \%(17 \%-33 \%) & \text { Beta } & \text { EINSTEIN-DVT }^{15} \\ \text { DVT } & 0.0 \% & - & \text { Assumption } \\ \text { Intracranial bleeding } & 43.6 \%(36.5 \%-50.7 \%) & \text { Beta } & \text { Linkins et al }^{21} \\ \text { Major extracranial bleeding } & 3.9 \%(2.7 \%-5.4 \%) & \text { Beta } & {\text { Linkins et } a l^{21}}^{24} \\ \text { CTEPH (3-year mortality) } & 26.0 \%(22 \%-30 \%) & \text { Beta } & {\text { Condliffe et } a l^{24}}\end{array}$

Utility scores

\begin{tabular}{|c|c|c|c|}
\hline Population norm & $0.929(0.917-0.941)$ & Beta & Guan and Liü25 \\
\hline DVT & $0.884(0.674-1.000)$ & Beta & Locadia et $a{ }^{26}$ \\
\hline $\mathrm{PE}$ & $0.663(0.379-0.905)$ & Beta & Locadia et $\left.a\right|^{26}$ \\
\hline Major extracranial bleeding & $0.684(0.516-0.905)$ & Beta & Locadia et $\left.a\right|^{26}$ \\
\hline CRNM bleeding & 1.000 & Beta & Assumption \\
\hline CTEPH & $0.560(0.528-0.592)$ & Beta & Meads et $a l^{29}$ \\
\hline Mild PTS & $1.000(0.91-1.00)$ & Beta & Lenert and Soetikno ${ }^{30}$ \\
\hline Severe PTS & $0.93(0.76-1.00)$ & Beta & Lenert and Soetikno ${ }^{30}$ \\
\hline Warfarin (disutility) & $0.988(0.95-1.00)$ & Beta & Marchetti et $\left.a\right|^{31}$ \\
\hline Enoxaparin (disutility) & $0.988(0.95-1.00)$ & - & Assumption \\
\hline Rivaroxaban (price/20 mg tablet) & $5.19(3.63-6.75)$ & - & \multirow{3}{*}{$\begin{array}{l}\text { Integrated } \\
\text { Management Platform } \\
\text { of Beijing Medicine } \\
\text { Sunshine Purchase }^{14}\end{array}$} \\
\hline Warfarin (price/3 mg tablet/day) & $0.08(0.06-0.10)$ & - & \\
\hline Enoxaparin (6000 units: $0.6 \mathrm{~mL}$ ) & $8.71(6.10-11.32)$ & - & \\
\hline
\end{tabular}

Monitoring cost (US\$)

$\begin{array}{llll}\text { Warfarin monitoring (per time) } & 10.98(7.69-14.27) & \text { Gamma } & \text { Local charge } \\ \text { Rivaroxaban monitoring (per time) } & 10.98(7.69-14.27) & \text { Gamma } & \text { Assumption }\end{array}$


Table 1 Continued

\begin{tabular}{|c|c|c|c|}
\hline & Base case (lower-upper) & Distribution & Source \\
\hline \multicolumn{4}{|l|}{ Costs of events (US\$) } \\
\hline rVTE-DVT & 3853 (2697-5009) & Gamma & Li et $a l^{33}$ \\
\hline CRNM bleeding & $8.25(5.77-10.72)$ & Gamma & Wu et $a l^{34}$ \\
\hline Major bleeding (extracranial) & 2999 (2099-3898) & Gamma & Wu et $a l^{34}$ \\
\hline Postintracranial bleeding & $339.6(237.7-441.5)$ & Gamma & Wu et $a l^{34}$ \\
\hline Mild/moderate PTS & 59.97 (41.98-77.96) & Gamma & Chen et $a l^{35}$ \\
\hline Severe PTS & $487.3(341.1-633.4)$ & Gamma & Chen et $a l^{35}$ \\
\hline СTEPH & $4873(3411-6334)$ & Gamma & Chen et $a l^{35}$ \\
\hline Frequency of monitoring - rivaroxaban & $3(2.1-3.9)$ & Gamma & Assumption \\
\hline $\begin{array}{l}\text { Length of stay of patients-enoxaparin/ } \\
\text { warfarin }\end{array}$ & $14.6(10.22-18.98)$ & Gamma & Wu et al ${ }^{7}$ \\
\hline $\begin{array}{l}\text { Difference in length of stay of patients- } \\
\text { rivaroxaban versus enoxaparin/warfarin }\end{array}$ & $3(2.1-3.9)$ & Gamma & van Bellen et $\left.a\right|^{32}$ \\
\hline
\end{tabular}

CRNM, clinically relevant non-major; CTEPH, chronic thromboembolic pulmonary hypertension; DVT, deep vein thrombosis; PE, pulmonary embolism; PTS, post-thrombotic syndrome; rVTE, recurrent venous thromboembolism.

incremental costs and QALYs. All analyses were carried out using Microsoft Excel.

Patient and Public Involvement

Patients were not involved.

\section{RESULTS}

\section{Base-case analysis}

The results of the base-case cost-effectiveness analysis are presented in table 2. Treatment with rivaroxaban and enoxaparin/warfarin over a 3-month period, estimated for a time duration of 5 years, showed that rivaroxaban therapy was associated with a gain of 0.008 QALYs, (4.111 QALYs with rivaroxaban compared with 4.103 QALYs with enoxaparin/warfarin). Although the drug acquisition cost of rivaroxaban was higher compared with enoxaparin/warfarin (US $\$ 504.9$ vs US $\$ 145.8$; difference of US\$359.0), the monitoring cost (US\$24.3 vs US $\$ 64.3$; difference of US\$ -40.0 ) and treatment cost for VTE events (US\$3625.2 vs US $\$ 4770.8$; difference of US\$ -1145.5) with rivaroxaban were lower compared with those for enoxaparin/warfarin. This resulted in an overall lower total cost of treatment with rivaroxaban than with enoxaparin/warfarin (US\$4744.4 vs US\$5572.4,

\begin{tabular}{|c|c|c|c|}
\hline Outcomes & Rivaroxaban & Enoxaparin/warfarin & Incremental \\
\hline Total cost (US\$) & 4744.4 & 5572.4 & -828.0 \\
\hline Drug acquisition cost & 504.9 & 145.8 & 359.0 \\
\hline Monitoring cost & 24.3 & 64.3 & -40.0 \\
\hline VTE event treatment cost & 3625.2 & 4770.8 & -1145.5 \\
\hline Bleeding treatment cost & 33.8 & 33.7 & 0.1 \\
\hline PTS/CTEPH & 556.1 & 557.8 & -1.6 \\
\hline QALY & 4.111 & 4.103 & 0.008 \\
\hline ICER & - & - & Dominant \\
\hline
\end{tabular}

CTEPH, chronic thromboembolic pulmonary hypertension; ICER, incremental cost-effectiveness ratio; PTS, post-thrombotic syndrome; QALY, quality-adjusted life year; VTE, venous thromboembolism. 


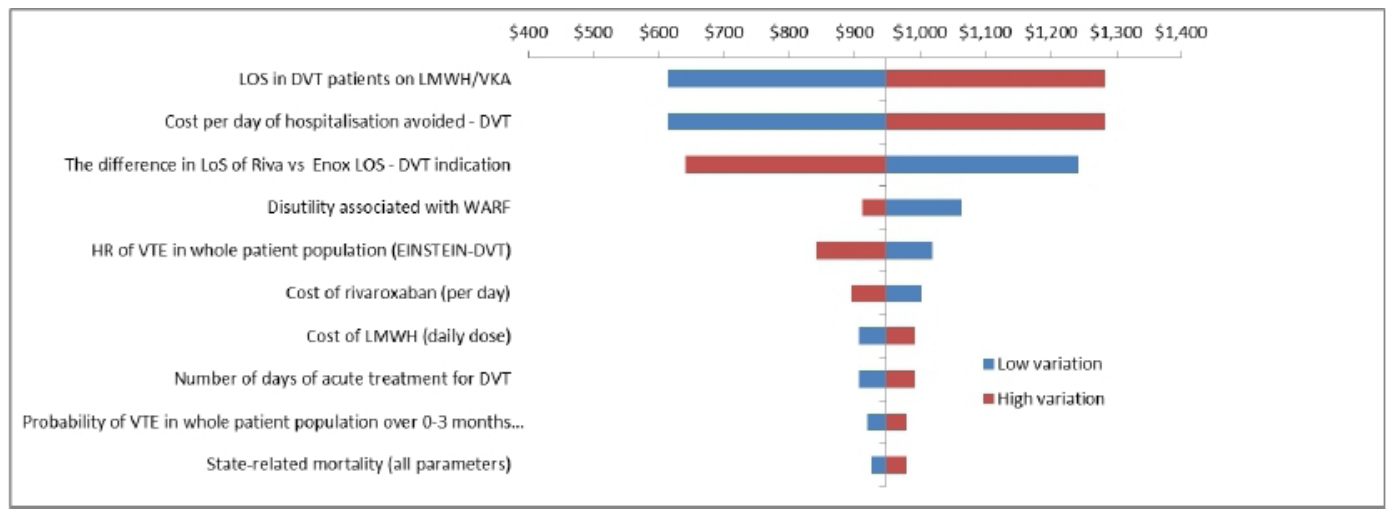

Figure 2 One-way sensitivity analysis tornado diagram for rivaroxaban compared with standard of care (net monetary benefit, quality-adjusted life year based). DVT, deep vein thrombosis; Enox, enoxaparin; LMWH, low-molecular weight heparin; LoS, length of stay; Riva, rivaroxaban; VKA, vitamin-K-antagonists; VTE, venous thromboembolism; WARF, warfarin.

respectively; incremental costs US\$-828.0). The cost of treating bleeding events, PTS and CTEPH were similar with both treatments and did not impact the overall cost of treatment (table 2).

\section{One-way sensitivity analysis}

Since rivaroxaban was dominant in the base-case analysis, a net monetary benefit (NMB) OWSA was conducted to examine economic value. The top 10 most sensitive parameters affecting the rivaroxaban and enoxaparin/ warfarin cost-effectiveness model are presented in figure 2. According to the OWSA, the cost-effectiveness of rivaroxaban compared with enoxaparin/warfarin was most sensitive to the length of hospital stay of patients on enoxaparin/warfarin, cost per day of hospitalisation and the difference in LoS between patients receiving rivaroxaban and enoxaparin/warfarin; these parameters acted as the main drivers of the cost differences. Overall, rivaroxaban showed a positive NMB irrespective of the parameters or the values used.

\section{Probabilistic sensitivity analysis}

The PSA confirmed the cost-effectiveness of rivaroxaban over enoxaparin/warfarin (figure 3). The majority of simulations showed that 3 months of treatment with

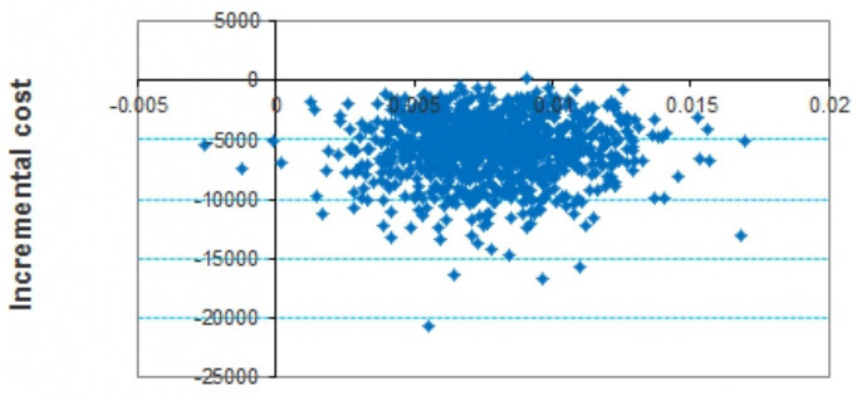

Incremental QALY

Figure 3 Cost-effectiveness plane for rivaroxaban versus enoxaparin/warfarin, based on whole study HR (5-year, QALY outcome). QALY, quality-adjusted life year. rivaroxaban was more cost-effective than the equivalent duration of enoxaparin/warfarin treatment, which resulted in a $99.6 \%$ likelihood of rivaroxaban being costeffective at a WTP threshold of US\$14 992.5 per QALY.

\section{DISCUSSION}

This study was an economic evaluation of rivaroxaban anticoagulation therapy compared with SoC for DVT treatment from a Chinese healthcare payer perspective. From the base-case analysis, it was observed that, over a 5-year period, rivaroxaban appeared to be more costeffective than SoC for the treatment of hospitalised acute DVT in China despite having a higher price per unit than warfarin. These results were mainly driven by the lower hospitalisation cost of patients receiving rivaroxaban. The sensitivity analyses also showed the robustness of the model used.

Our findings show that hospitalisation costs for monitoring and VTE-related events were lower with rivaroxaban compared with SoC treatment. Although only 0.008 additional QALYs were achieved with rivaroxaban treatment, the PSA suggested that the probability of rivaroxaban being more cost-effective than SoC treatment would be $99.6 \%$ per 1000 iterations, indicating that rivaroxaban has greater cost-saving potential than enoxaparin/ warfarin, at a WTP threshold of US\$14 992.5 per QALY.

The results of our study are in line with those presented in the previous studies. Studies in the Western population have demonstrated the cost-effectiveness of rivaroxaban over LMWH/VKA, placebo, LMWH alone and VKA alone for VTE recurrence and other transition events. ${ }^{19}{ }^{38-40}$ In a cost-effectiveness analysis, rivaroxaban showed per-patient cost savings at 3, 6 and 12 months compared with enoxaparin/warfarin in the EINTEIN DVT trial; the HR of VTE, discount rate and mean age were the driving factors affecting this model. ${ }^{19}$ Coleman et al showed greater QALYs gained with rivaroxaban treatment compared with placebo (16.167 vs 16.134) despite a higher treatment cost (US\$22 645 vs US\$22 083), suggesting the higher cost-effectiveness of rivaroxaban 
over placebo, assuming a WTP threshold of US\$50 000 per QALYs gained. ${ }^{38}$ An economic comparison of rivaroxaban and warfarin in the US showed a lower cost of treatment with rivaroxaban (US\$3195 vs US\$6188) as well as more QALYs gained (9.29 QALYs vs 9.14 QALYs). However, rivaroxaban was not more cost-effective than warfarin when major bleeding risk with rivaroxaban exceeded 3.8\%. ${ }^{39}$ Gourzoulidis et al reported the costeffectiveness analysis of rivaroxaban for VTE treatment in Greece from a third-party payer perspective, which also showed that rivaroxaban was cost-effective compared with SoC. ${ }^{40}$ The findings from all these studies suggest that treatment with rivaroxaban results in greater cost benefits and clinical outcomes from both payer and societal perspectives.

The findings of our study imply that, despite the cost of rivaroxaban being higher than that of warfarin, it has the potential to reduce the overall economic burden of DVT treatment by reducing hospitalisation costs. This is particularly meaningful for the Chinese healthcare system and its hospitals and payers, who are struggling to reduce patient LoS and healthcare expenses. ${ }^{41}$ With rivaroxaban, patients may have higher utility and satisfaction as well as lower economic burden due to early discharge and convenient disease management methods. However, the duration of anticoagulation and patients' age must be important consideration, as in the previous study, recurrence of VTE was associated with shorter duration of anticoagulation, older age and primary DVT. ${ }^{20}$

Although methodological standards were followed for the conduct of this analysis, it has several limitations. First, we set a lot of assumptions in the model which may not reflect real-world clinical practice, for example, all patients were receiving inpatient treatment and the anticoagulant duration was only 3 months with frequent monitoring visits. We then extrapolated the results to wider populations, focusing on the high impact of hospitalisations. Second, clinical and utility data were derived from many sources, some of which were not specific to the Chinese population. For example, the clinical inputs on efficacy and safety were taken from the EINSTEIN-DVT trial, and some of the utilities data came from international literature because of a lack of Chinese-specific sources; therefore, further validation is warranted before applying these findings in real-world treatment settings. However, including the limited economic data available from China were the best possible measure taken to address the concern. Third, our model lacked analyses based on patient/societal perspectives, which may also be beneficial in evaluating the indirect cost of rivaroxaban treatment. Real-world studies would also be useful to evaluate the actual cost-effectiveness of rivaroxaban and further justify its clinical and economic values.

\section{CONCLUSION}

In conclusion, our study showed rivaroxaban to be a costsaving treatment option when compared with enoxaparin/ warfarin therapy for hospitalised acute DVT treatment in Chinese patients. The sensitivity of the cost-effectiveness model was mainly driven by the LoS of patients on enoxaparin/warfarin treatment, cost per day of hospitalisation and the difference in LoS of rivaroxaban-treated and enoxaparin/warfarin-treated patients.

Acknowledgements The authors thank Karan Sharma and Dr Amit Bhat from Indegene, Bangalore, for providing necessary medical writing assistance.

Contributors LY contributed to the design, interpretation of the data and revisions and JW contributed to the modelling and drafting the manuscript.

Funding This study was funded by National Natural Science Foundation of China (Grant numbers 71273016/G0308 and 71673004/G0406). The data collection was sponsored by Bayer Healthcare Company; however, publication of the study results was not contingent on the sponsor's approval.

Competing interests LY has nothing to disclose. JW is an employee of Bayer Healthcare Company Ltd.

Patient consent for publication Not required.

Ethics approval All procedures performed in studies involving human participants were in accordance with the ethical standards of the ethical committee of Peking University Health Science Center (IRB00001052-17006) and with the 1964 Helsinki declaration and its later amendments or comparable ethical standards.

Provenance and peer review Not commissioned; externally peer reviewed.

Data availability statement All data relevant to the study are included in the article.

Open access This is an open access article distributed in accordance with the Creative Commons Attribution Non Commercial (CC BY-NC 4.0) license, which permits others to distribute, remix, adapt, build upon this work non-commercially, and license their derivative works on different terms, provided the original work is properly cited, appropriate credit is given, any changes made indicated, and the use is non-commercial. See: http://creativecommons.org/licenses/by-nc/4.0/.

ORCID iD

Li Yang http://orcid.org/0000-0003-0640-2003

\section{REFERENCES}

1 Silva AS, Brazao ML, Granito S, et al. Thrombophilia/prothrombotic disorders. Sociedade Portuguesa de Medicina interna 2010;17:44-58.

2 Cheng G, Chan C, Liu YT, et al. Incidence of deep vein thrombosis in hospitalized Chinese medical patients and the impact of DVT prophylaxis. Thrombosis 2011;2011:629383

3 Yang Y, Liang L, Zhai Z, et al. Pulmonary embolism incidence and fatality trends in Chinese hospitals from 1997 to 2008: a multicenter registration study. PLoS One 2011;6:e26861.

4 Law Y, Chan YC, Cheng SWK. Epidemiological updates of venous thromboembolism in a Chinese population. Asian J Surg 2018;41:176-82.

5 Joynt GM, Li TST, Griffith JF, et al. The incidence of deep venous thrombosis in Chinese medical intensive care unit patients. Hong Kong Med J 2009;15:24-30.

6 Kearon C, Akl EA, Ornelas J, et al. Antithrombotic therapy for VTe disease: chest guideline and expert panel report. Chest 2016;149:315-52.

7 Wu EQ, Xie J, Wu C, et al. Treatment, monitoring, and economic outcomes of venous thromboembolism among hospitalized patients in China. Pharmacoeconomics 2014;32:305-13.

8 Kubitza D, Becka M, Voith B, et al. Safety, pharmacodynamics, and pharmacokinetics of single doses of Bay 59-7939, an oral, direct factor Xa inhibitor. Clin Pharmacol Ther 2005;78:412-21.

9 Eriksson BI, Kakkar AK, Turpie AGG, et al. Oral rivaroxaban for the prevention of symptomatic venous thromboembolism after elective hip and knee replacement. J Bone Joint Surg Br 2009;91:636-44.

10 Turpie AGG, Lassen MR, Davidson BL, et al. Rivaroxaban versus enoxaparin for thromboprophylaxis after total knee arthroplasty (RECORD4): a randomised trial. Lancet 2009;373:1673-80.

11 Wang Y, Wang C, Chen Z, et al. Rivaroxaban for the treatment of symptomatic deep-vein thrombosis and pulmonary embolism in 
Chinese patients: a subgroup analysis of the Einstein DVT and PE studies. Thromb J 2013;11:25.

12 Deitelzweig S, Laliberté F, Crivera C, et al. Hospitalizations and other health care resource utilization among patients with deep vein thrombosis treated with rivaroxaban versus low-molecularweight heparin and warfarin in the outpatient setting. Clin Ther 2016;38:1803-16.

13 Merli GJ, Hollander JE, Lefebvre P, et al. Rates of hospitalization among patients with deep vein thrombosis before and after the introduction of rivaroxaban. Hosp Pract 2015;43:85-93.

14 Integrated Management Platform of Beijing Medicine Sunshine Purchase. Unit costs of medicine. Available: http://210.73.89.76/ ServiceSelect/GetServiceSelectList [Accessed 6 Nov 2017].

15 Bauersachs R, Berkowitz SD, et al, EINSTEIN Investigators. Oral rivaroxaban for symptomatic venous thromboembolism. N Engl J Med 2010;363:2499-510.

16 Lefebvre P, Coleman Cl, Bookhart BK, et al. Cost-Effectiveness of rivaroxaban compared with enoxaparin plus a vitamin $\mathrm{K}$ antagonist for the treatment of venous thromboembolism. J Med Econ 2014;17:52-64.

17 Husereau D, Drummond M, Petrou S, et al. Consolidated health economic evaluation reporting standards (cheers) statement. Value Health 2013:16:231-50.

18 Wang $\mathrm{H}$, Ye J, Wang L, et al. Risk characteristics of venous thromboembolism in Chinese patients. Clin Appl Thromb Hemost 2016;22:490-4.

19 Bamber L, Muston D, McLeod E, et al. Cost-Effectiveness analysis of treatment of venous thromboembolism with rivaroxaban compared with combined low molecular weight heparin/vitamin $\mathrm{K}$ antagonist. Thromb J 2015;13:20.

20 Prandoni P, Noventa F, Ghirarduzzi A, et al. The risk of recurrent venous thromboembolism after discontinuing anticoagulation in patients with acute proximal deep vein thrombosis or pulmonary embolism. A prospective cohort study in 1,626 patients. Haematologica 2007;92:199-205.

21 Linkins L, O'Donnell M, Julian JA, et al. Intracranial and fatal bleeding according to indication for long-term oral anticoagulant therapy. $J$ Thromb Haemost 2010;8:2201-7.

22 Miniati M, Monti S, Bottai M, et al. Survival and restoration of pulmonary perfusion in a long-term follow-up of patients after acute pulmonary embolism. Medicine 2006;85:253-62.

23 Prandoni P, Villalta S, Bagatella P, et al. The clinical course of deep-vein thrombosis. prospective long-term follow-up of 528 symptomatic patients. Haematologica 1997;82:423-8.

24 Condliffe R, Kiely DG, Gibbs JSR, et al. Improved outcomes in medically and surgically treated chronic thromboembolic pulmonary hypertension. Am J Respir Crit Care Med 2008;177:1122-7.

25 Guan H, Liu G. Comparison analysis on health related quality of life among urban and rural residents in 4 cities of China. Chinese Health Econ 2015;34:5-12.

26 Locadia M, Bossuyt PMM, Stalmeier PFM, et al. Treatment of venous thromboembolism with vitamin $\mathrm{K}$ antagonists: patients' health state valuations and treatment preferences. Thromb Haemost 2004;92:1336-41.

27 Bamber L, Wang MY, Prins MH, et al. Patient-Reported treatment satisfaction with oral rivaroxaban versus standard therapy in the treatment of acute symptomatic deep-vein thrombosis. Thromb Haemost 2013;110:732-41.

28 Rivero-Arias O, Ouellet M, Gray A, et al. Mapping the modified Rankin scale (mRS) measurement into the generic EuroQol (EQ-5D) health outcome. Med Decis Making 2010;30:341-54.

29 Meads DM, McKenna SP, Doughty N, et al. The responsiveness and validity of the camphor utility index. Eur Respir J 2008;32:1513-9.

30 Lenert LA, Soetikno RM. Automated computer interviews to elicit utilities: potential applications in the treatment of deep venous thrombosis. J Am Med Inform Assoc 1997:4:49-56.

31 Marchetti M, Pistorio A, Barone M, et al. Low-Molecular-Weight heparin versus warfarin for secondary prophylaxis of venous thromboembolism: a cost-effectiveness analysis. Am J Med 2001;111:130-9.

32 van Bellen B, Bamber L, Correa de Carvalho F, et al. Reduction in the length of stay with rivaroxaban as a single-drug regimen for the treatment of deep vein thrombosis and pulmonary embolism. Curr Med Res Opin 2014;30:829-37.

33 QY L, YC L, Zhang YQ. Analysis of clinical data of 1136 inpatients with venous thromboembolism in 2003-2013. J Pract Med 2015;6:1006-8.

34 Wu B, Kun L, Liu X, et al. Cost-Effectiveness of different strategies for stroke prevention in patients with atrial fibrillation in a health resource-limited setting. Cardiovasc Drugs Ther 2014;28:87-98.

35 Chen X, Wang C, Zhu M. Cost-Effectiveness study of rivaroxaban for the preverention of venous thromboembolism in patients undergoing total knee replacement. China Pharmacy 2011;30:2787-90.

36 The World Bank. GDP per capita (current US\$).Washington, DC, 2016. Available: http://data.worldbank.org/indicator/NY.GDP.PCAP. CD [Accessed 20 Jul 2018].

37 China guidelines for pharmacoeconomic evaluations. Beijing: China center for health economics research, 2016.

38 Coleman Cl, Limone BL, Bookhart BK, et al. Cost-Effectiveness analysis of extended duration anticoagulation with rivaroxaban to prevent recurrent venous thromboembolism. Thromb Res 2014;133:743-9.

39 Seaman CD, Smith KJ, Ragni MV. Cost-Effectiveness of rivaroxaban versus warfarin anticoagulation for the prevention of recurrent venous thromboembolism: a U.S. perspective. Thromb Res 2013;132:647-51.

40 Gourzoulidis G, Kourlaba G, Kakisis J, et al. Cost-Effectiveness analysis of rivaroxaban for treatment of deep vein thrombosis and pulmonary embolism in Greece. Clin Drug Investig 2017;37:833-44.

41 Pan X, Dib HH, Zhu M, et al. Absence of appropriate hospitalization cost control for patients with medical insurance: a comparative analysis study. Health Econ 2009;18:1146-62. 